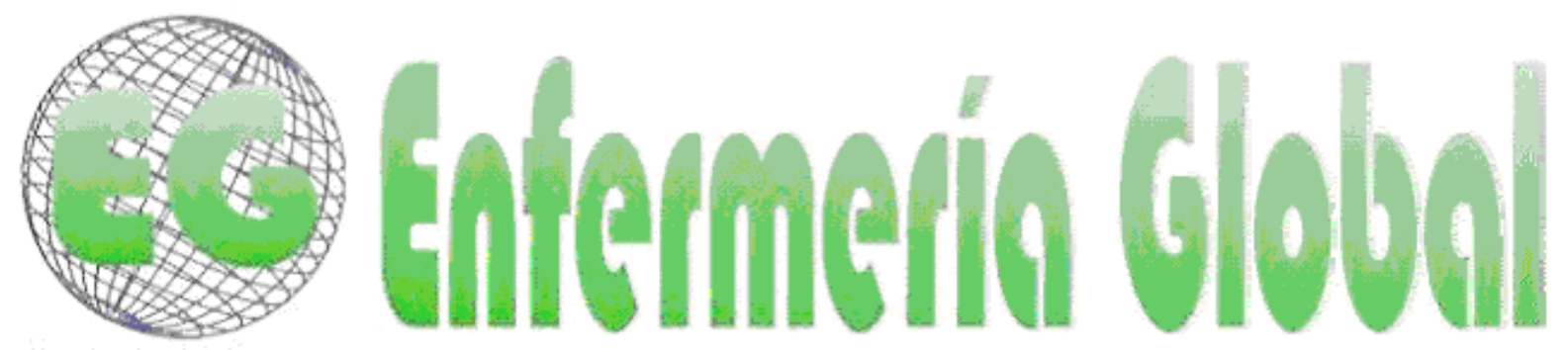

\title{
Aproximación a la Ablación/Mutilación Genital Femenina (A/MGF) desde la Enfermería Transcultural. Una revisión bibliográfica.
}

Approximation to the Ablation/Female Genital Mutilation (A/FGM) from the Transcultural Nursing. A bibliographical revisión.

\section{*Jiménez Ruiz, I. **Almansa Martínez, P. *Pastor Bravo, MM. **Pina Roche, F.}

"Graduado/a en Enfermería. Máster en "Salud, Mujer y Cuidados" . E-mail: ismaeljr1990@gmail.com **Profesora Facultad de Enfermería. Universidad de Murcia.

Palabras clave: ablación/mutilación genital femenina; ablación, circuncisión femenina; enfermería transcultural.

Keywords: ablation/female genital mutilation, ablation, female circumcision, and transcultural nursing.

\section{RESUMEN}

Objetivo: Analizar las argumentaciones favorables a la Ablación/Mutilación Genital Femenina (A/MGF) con el fin de conocer y comprender la realidad compleja y subjetiva de la A/MGF.

Material y método: Búsqueda y revisión bibliográfica en la red para acceder a directorios Web de organizaciones, y en las principales bases de datos de ciencias de la salud.

Resultados: El análisis de la bibliografía aporta una gran cantidad de información acerca de la fundamentación de la práctica y de las complicaciones que derivan de la misma, esclareciendo por tanto las complejas situaciones que se dan a la hora de perpetuarla.

\section{Conclusiones:}

La A/MGF es entendida como un cuidado cultural de las mujeres determinado por factores socioculturales, higiénico-estéticos, religioso-espirituales, sexuales y otros factores relacionados con la salud. Asimismo, se ha descrito una amplia gama de complicaciones secundarias a la A/MGF.

\section{ABSTRACT}

Objective: Analysing the argumentations in favour to the Female Genital Mutilation (FGM) with the intention to know and understand the complex and subjective reality of this practice. 
Material and method: bibliographical search and revision on the net in order to accessing Web directories of organizations and the main health sciences data bases.

Results: The analysis of the biography contributes with a big quantity of information regarding the supportiveness of this practice and the complications derived from it, clarifying the complex situations involved in its perpetuation.

Conclusions: The FGM is understood as a cultural care of women determined by socio-cultural, hygienic- aesthetic, religious- spiritual and sexual factors among others related with health. In this way, a wide range of secondary complications to FGM have been described.

\section{INTRODUCCIÓN}

La ablación/mutilación genital femenina (A/MGF), es definida por la OMS ${ }^{(1)}$ como todos aquellos procedimientos consistentes en la resección parcial o total de los genitales femeninos externos, así como otras lesiones de los órganos genitales femeninos, por motivos no médicos.

Esta práctica es la más extendida y practicada dentro del grupo de costumbres tradicionales perjudiciales, ya que actualmente, estimaciones basadas en datos de prevalencia recientes, indican que 91,5 millones de niñas y mujeres por encima de 9 años de edad en África viven bajo las consecuencias de la A/MGF. También se estima que 3 millones de niñas en África corren el riesgo de someterse a la mutilación genital femenina cada año ${ }^{(2)}$.

A día de hoy, el contexto geográfico en el que se realiza la práctica de forma habitual abarca unos 28 países del África subsahariana, y es también habitual en algunos países de Oriente Medio como Egipto, Yemen o los Emiratos Árabes ${ }^{(3)}$. Asimismo, documentos recientes respaldan la hipótesis de que la A/MGF se realiza de forma habitual en el noreste de Sudáfrica ${ }^{(4)}$.

Del mismo modo, es necesario subrayar que como consecuencia de los movimientos migratorios, ha comenzado a afectar a países receptores de población procedente de estas zonas, como es el caso de España ${ }^{(5)}$. Hecho que ha creado, en algunos sectores sociales y profesionales, la necesidad de aprender a abordar esta problemática desde una perspectiva de protección de la dignidad y los derechos humanos de las personas.

Por lo tanto, la importancia de profundizar en este tema reside principalmente en que es una práctica que atenta contra la integridad biopsicosocial tanto de mujeres como de las niñas, pudiendo llegar a producir graves complicaciones físicas, obstétricas y/o psicosociales. Además supone una vulneración de la dignidad y de los derechos humanos de las mujeres y las niñas, en tanto que transgrede su derecho a la vida, a la libertad y a la seguridad de su persona, ayudando de este modo a la preservación de las relaciones de dominación-sumisión entre hombres y mujeres que rigen las sociedades patriarcales.

Entendemos también que el abordaje de la prevención de la A/MGF desde los servicios públicos de salud, en general, y la Enfermería en particular, supone un importante y dificultoso reto, debido a las connotaciones culturales tan arraigadas en los países donde se realiza y a que el paradigma biomédico no responde a ciertas necesidades y desafíos que propone, la ya establecida, población multicultural y 
globalizada.

Asimismo, creemos que la Teoría de la Diversidad y de la Universalidad de los Cuidados Culturales de Madeleine Leininger, es la teoría enfermera más apropiada para conceptualizar esta revisión bibliográfica, puesto que la competencia transcultural de los/las profesionales sanitarios es la que va a determinar la capacidad de gestionar cuidados en el ámbito de la prevención de la A/MGF, ya que solamente estos cuidados transculturales garantizarán la superación de la limitación que supone una visión etnocentrista del mundo y por tanto de las intervenciones enfermeras.

Así pues el objetivo que se persigue, con la presente revisión de la literatura relativa a la A/MGF, es analizar las fundamentaciones de la A/MGF a través de la Enfermería Transcultural con el fin de conocer y comprender la realidad compleja y subjetiva de la ablación/mutilación genital femenina.

\section{MATERIAL Y MÉTODO}

Se realizó una revisión bibliográfica, entre noviembre de 2011 y febrero de 2012, con el fin de proporcionar una comprensión general de las variables que posteriormente fueron analizadas. Mediante la búsqueda y revisión bibliográfica se desarrolló una extensa contextualización a partir de la cual examinar las situaciones humanas complejas que derivan de la confluencia cultural entre la forma de ver el mundo occidental y la de los países en los que se practica la A/MGF.

Al inicio de la búsqueda bibliográfica se realizó una somera revisión en motores de búsqueda en red del tipo Google y Google Académico, a través de los cuales se accedió a diferentes documentos, de gran interés para el estudio, existentes en los directorios Web de organizaciones como Amnistía Internacional (Al), Comité Interafricano, Grupo Interdisciplinar para la prevención y estudio de las Prácticas Tradicionales Perjudiciales (GIP/PTP), United Nations (UN), United Nations High Commisioner for Regugees (UNHCR), Organización Mundial de la Salud (OMS), United Nations International Children's Emergency Fund (UNICEF), Mujer Inmigrante y Asistencia Sanitaria (MIAS), Women's Islamic Initiative in Spirituality and Equality (WISE), Grupo Interdisciplinar de Estudios sobre Migraciones, Interculturalidad y Ciudadanía (GIEMIC) y Unión de Asociaciones Familiares (UNAF). Posteriormente se procedió a realizar una búsqueda más exhaustiva en las bases de datos: CINAHL, Cochrane Library Plus, CUIDEN, DOAJ, MEDLINE, SciELO y Web of Knowledge.

La búsqueda en bases de datos se ha realizado en dos grandes bloques, con resultados dispares. El primer bloque se corresponde con la contextualización de la A/MGF en la teoría aplicada. Con el segundo bloque de búsqueda se ha pretendido profundizar en las diferentes características de la práctica y comprobar la existencia y rigurosidad de las fuentes primarias relacionadas con la fundamentación y consecuencias de la A/MGF.

Los descriptores en español que se utilizaron para la búsqueda han sido una combinación de los siguientes: Madeleine Leininger, Enfermería Transcultural, Etnoenfermería, mutilación genital femenina, circuncisión femenina, ablación, clitoridectomía, escisión, infibulación, Enfermería. En cuanto a los descriptores en inglés fue una combinación de: Madeleine Leininger, Transcultural Nursing, 
Ethnonursing, female genital mutilation, female circumcision, ablation, clitoridectomy, escisión, infibulation y Nursing.

\section{RESULTADOS}

Los datos extraídos de la revisión fueron agrupados en dos grandes categorías con sus respectivos subgrupos.

\section{Sistema de cuidados genéricos tradicionales y dimensiones culturales de la A/MGF.}

La A/MGF, como sistema de cuidados genéricos tradicionales, es entendida como creencias regidas por modelos dinámicos de interrelación de factores estructurales de la comunidad, como pueden ser los valores religiosos, socio-culturales, sexuales, higiénico-estéticos o de salud.

A la hora de contextualizar esta práctica es necesario tener un especial cuidado en no incurrir ni en el etnocentrismo ni en el relativismo cultural más radical. A pesar de que la comprensión occidental de lo que está bien o está mal solamente puede tiene vigor en nuestro contexto socio-cultural, bien es cierto que la cuestión de la A/MGF no es algo que podamos ignorar, ya que colisiona con el desarrollo de las mujeres y está fundamentada y consolidada en valores afianzados desde una sociedad marcadamente androcentrista y desigualitaria.

\subsection{Factores socio-culturales.}

En algunas comunidades, la A/MGF se realiza como parte de un rito de iniciación con fuertes connotaciones simbólicas para la comunidad. Este proceso de paso a la primera etapa de la adultez no se le realiza únicamente en la mujer, ya que se efectúa en ambos sexos. En lengua mandinga (hablada mayoritariamente en Gambia y de amplia difusión en Guinea Bissau, Malí (bambara), Sierra Leona (mandé) y al sur de Senegal) el proceso de iniciación masculino recibe el nombre de kaseo y el femenino ñyakaa, en ambos casos el significado es el mismo y presentan tres fases: separación, marginación y agregación como afirman Adriana Kaplan y María Bedoya $^{(11)}$.

Kaplan y Bedoya ${ }^{(11)}$ describen las fases del rito de iniciación de la siguiente forma:

"En la primera fase, llamada de separación, los/las niños/as son alejados/as de la comunidad y circuncidados/as. Se produce pues una ruptura con la etapa de la infancia, esta ruptura con la etapa anterior está marcada por el corte del prepucio o la escisión del clítoris, la sangre y el dolor.

La segunda fase, denominada de marginación, tiene un tiempo de duración muy variable, pues depende principalmente del tiempo de cicatrización de la herida. El tiempo de curación de la circuncisión oscila entre dos y tres semanas. La cicatrización de la herida producida en las mujeres depende del tipo de intervención que se le realice, variando desde dos semanas en una clitoridectomía, a ochos semanas en casos de infibulación. Esta fase es la más crítica pues en ella se dan las complicaciones agudas de la operación. Además es un período en el que se separa a los/las niños/as de la comunidad y está rodeado de tabúes, normas, prescripciones y prohibiciones especiales que irán 
configurando las señas de identidad propias del nuevo miembro de la comunidad. Se crean así una serie de marcas físicas imborrables como consecuencia del "corte" y una serie de conductas difícilmente alterables relacionadas con la identidad étnica y de genero.

Durante la tercera fase, de agregación, se realiza una fiesta en la que se presenta a los nuevos miembros, con sus nuevos roles y sus nuevas categorías sociales. Son pues reconocidas socialmente por su comunidad. Las mujeres pasan a formar parte del mundo de las mujeres y los hombres a formar parte del grupo de los hombres".

También es cierto que las sociedades que realizan este tipo de actos son marcadamente patriarcales, en las que el acceso de la mujer a la seguridad o a la tierra depende únicamente del casamiento con un hombre. La ablación por lo tanto es el factor determinante para crear la virtud de una mujer. Esta será decente y elegible para el matrimonio si está circuncidada y todo lo contrario si no lo está. Por tanto la no realización de la práctica condena a la niña a una vida de exclusión y humillación, lo que lleva a las madres a seguir hacia delante con la práctica y facilitarles las opciones de vida a sus hijas. La A/MGF, en estas sociedades, pasa de ser un sacrificio de salud y placer sexual a un hecho inherente a su pertenencia a la comunidad y un factor de creación de tejido social.

\subsection{Factores higiénico-estéticos.}

Otras de las razones a las que acuden quienes defienden la práctica son las higiénicas. En países como Egipto, Malí o Sudán, las mujeres que mantienen íntegros sus genitales son mujeres sucias y por lo tanto no se les permite manipular agua ni alimentos $^{(11)}$, "Dicen que de una solima (mujer no circuncidada) no se puede beber agua ni ingerir alimentos que haya manipulado, porque a través de su estado de impureza, ejerce una acción contaminante sobre aquello que toca". Por lo tanto, no se considera ni a un hombre ni a una mujer limpios hasta que se produce la ablación del prepucio o del clítoris, respectivamente. Así encontramos entre diversos grupos étnicos que en su lenguaje popular se refieren a la A/MGF con términos que tienen un significado de purificación, tahara en Egipto o tahur en Sudán, o un significado de limpieza, como sili-ji entre los bambaras de Malí.

En cuanto a las razones estéticas, existe la percepción en algunas comunidades de que los genitales femeninos son feos y voluminosos. $Y$ en otras creen que los genitales de la mujer pueden crecer lo suficiente como para resultar molestos, por estar colgando entre las piernas.

\subsection{Factores religioso-espirituales.}

Aunque no existe ninguna relación directa entre la A/MGF y las religiones mayoritarias, hay cierta creencia dentro de algunas comunidades de que dicha práctica es condición necesaria para que las mujeres sean puras. En los países más industrializados, existe la creencia de que esta práctica está relacionada íntimamente con el Islam, aunque bien es cierto que se han descrito casos en población musulmana, cristiana, judía ortodoxa y animista.

La práctica es de origen pre-islámico y no es habitual en la mayoría de países musulmanes, como ocurre en Arabia Saudí, la cuna del Islam, donde se desconoce, 
aunque ha adquirido una dimensión religiosa en algunos países musulmanes donde se practica. A pesar de que en el Corán no aparezca ninguna alusión sobre la A/MGF, las argumentaciones que atribuyen a esta práctica una fundamentación islámica están basadas en varios hadices (dichos del profeta Mahoma), en los que se menciona la A/MGF, pero cuya autenticidad no puede ser confirmada. Uno de los más populares es: "No corte demasiado, ya que es mejor para la mujer y más deseable para un marido" ${ }^{\prime 12)}$.

De todos modos, aunque esos hadices tuvieran validez, se refieren al tipo más leve de A/MGF, y solo se trata de una recomendación pero de ningún modo una obligación (a diferencia de la circuncisión masculina que tiene un carácter preceptivo tanto para los varones musulmanes como para los varones judíos $\left.{ }^{(7)}\right)$. Cabe mencionar que entre las diferentes escuelas jurídicas no existe en consenso sobre la realización de esta práctica. Por ejemplo para la escuela malikî (actualmente en vigor en el Magreb) el jitân es una práctica obligatoria para los hombres mientras que el jifâD, recomendable (mukarimma) para las mujeres. Otros, como la escuela shâf $\hat{\imath}$, que rige actualmente en Bahrein, África Oriental y Egipto, dicen que es obligatoria (gâdjib) ${ }^{(13)}$.

Aunque hoy día se siga relacionando la A/MGF con motivos religiosos, bien es cierto que los discursos que guían estos razonamientos pierden cada vez más fuerza. Prueba de ello es la Primera Conferencia Islámica para la Infancia en Rabat (Marruecos), celebrada en 2005 y de la cual surgió la Declaración de Rabat, en la que se condenaron, como contrarias al Islam, tanto la mutilación genital femenina como otras prácticas nefastas que discriminan a las niñas ${ }^{(14)}$.

\subsection{Factores sexuales.}

El control de la sexualidad de las mujeres es uno de los principales motivos del razonamiento de la A/MGF. Como afirma Anika Rahman ${ }^{(15)}$, la sexualidad es construida socialmente de forma diferente en distintas comunidades. Así nos encontramos con que en gran parte de las comunidades donde se realiza esta práctica, se cree que reduce el deseo sexual de las mujeres garantizando así el mantenimiento de su virginidad o pureza y la fidelidad hacia el marido. En Egipto, Sudán, o Somalia, el honor de las familias depende de la virginidad de las niñas ${ }^{(15)}$, por lo que la A/MGF se les practica a estas para "protegerlas" de posibles relaciones prematrimoniales que pueden deshonrar tanto a las niñas como a la familia.

En otros contextos, como en Kenia, Mali y Uganda, donde se practica la poligamia y la pureza no es la mayor preocupación, la A/MGF se lleva a cabo para reducir las demandas sexuales de las mujeres hacia sus maridos y controlar el deseo sexual de las mismas con el fin de promover la virginidad y la fidelidad conyugal ${ }^{(15)}$, se persigue pues, en estos entornos, un interés marcadamente masculino por la preservación de la poligamia ante un miedo irracional a la voracidad sexual de las mujeres. Por último, cabe destacar que existe la creencia de que los tipos II y III de A/MGF contribuyen favorablemente en la salud del matrimonio, puesto que se cree que un introito más estrecho proporciona un mayor placer a los hombres durante las relaciones sexuales $^{(7)}$.

\subsection{Factores relacionados con la salud, A/MGF como Cuidado Cultural.}

En algunas culturas existe la creencia de que la no A/MGF puede tener consecuencias negativas para la salud, tanto para las propias mujeres que sufren esta 
práctica, como para sus maridos y descendencia, como demuestran los siguientes casos. En algunas etnias mantener relaciones sexuales con una mujer que no ha sido sometida a A/MGF puede llegar a ser mortal, ya que el contacto del pene con el clítoris puede desencadenar la muerte del varón ${ }^{(16)}$.

También es relativamente frecuente encontrar culturas en las que se afirma que la A/MGF facilita el parto, ya que el contacto del clítoris con el neonato puede provocar la muerte de este ${ }^{(16)}$.

Igualmente existen creencias de que la realización de esta práctica favorece la fertilidad de las mujeres, aunque como veremos a continuación, ninguna de estas consideraciones que relacionan la práctica de la A/MGF con la promoción de la salud tiene cabida desde un punto de vista científico.

\section{Consecuencias negativas para la salud de las mujeres.}

La A/MGF suele implicar una serie de pérdidas de salud reproductiva, física, psíquica y social de las mujeres. Las complicaciones asociadas a la A/MGF, se pueden clasificar en:

2.1) Físicas (inmediatas y largo plazo).

2.2) Obstétrico-ginecológicas.

2.3) Psico-sociales.

2.4) Sexuales.

\subsection{Físicas.}

Utilizaremos el término complicaciones físicas para designar a todas aquellas complicaciones asociadas a problemas puramente orgánicos asociados a la A/MGF, exceptuando a las pertenecientes a la categoría de obstétrico-ginecológicas. Dentro de las complicaciones físicas diferenciaremos entre inmediatas y a largo plazo como lo hace Adriana Kaplan en $2011^{(17)}$.

\subsubsection{Inmediatas}

Hablaremos de complicaciones inmediatas cuando aparezcan entre el momento en el que se realiza el corte y diez horas después. Podemos encontrar:

- Hemorragia: es una de las complicaciones más comunes. Se trata del sangrado excesivo de la zona perineal como consecuencia de la ejecución del corte. La zona perineal es una región muy vascularizada y por ende la sección de la zona puede producir un sangrado profuso inmediato, como consecuencia de la sección arterial (arteria clitoridea) y venosa, o secundario al desprendimiento de un coagulo ${ }^{(3)(17)(18)}$.

- Anemia aguda posthemorrágica: se trata de un síndrome caracterizado por la disminución del nivel de hematocrito por debajo de parámetros normales a consecuencia de la pérdida de sangre resultante de la $\mathrm{A} / \mathrm{MGF}^{(18)}$.

- Shock: existe una gran variedad de shocks relacionados con la A/MGF, entre ellos encontramos: el hipovolémico hemorrágico, como consecuencia del sangrado profuso que produce el corte; distributivo séptico, provocado por la 
pérdida masiva de líquido causado por la acción de potentes endotoxinas; y neurogénico causado por dolor agudo provocado por la incisión en la zona genital ${ }^{(18)}$.

- Dolor intenso: la gran cantidad de terminaciones nerviosas existentes en la región genital y el deficiente uso, en ocasiones nulo, de medidas anestésicas, hace que el corte provoque un fuerte dolor en la zona que irá disminuyendo durante el período de curación. Como podemos leer en el apartado anterior, el dolor puede llegar a ser tan importante que puede derivar en un shock neurogénico ${ }^{(3)(17)(18)}$.

- Infección ${ }^{(18)}$ : las invasiones por microorganismos patógenos derivadas de la A/MGF vienen determinada por las precarias condiciones de asepsia utilizadas en este tipo de intervenciones. Así podemos encontrar desde una simple infección local hasta una septicemia que ocasione la muerte de la mujer. Según un estudio de Adriana Kaplan en $2011^{(17)}$, de las complicaciones inmediatas asociadas a los tres tipos de A/MGF estudiados, la infección, es la más común junto a la hemorragia y la anemia. Las complicaciones potencialmente asociadas a la infección provocada por la A/MGF son: tétanos, enfermedades de transmisión sanguínea $\mathrm{VIH} / \mathrm{SIDA}$ y hepatitis $\mathrm{B}$ y $\mathrm{C}$, como consecuencia de la utilización del mismo material sin esterilizar para diferentes intervenciones en distintas niñas o mujeres. Cistitis o infecciones de la mucosa uretral, vulvovaginitis y septicemia.

- Lesiones en tejidos periféricos: como consecuencia de la dificultad de sujeción de las niñas/mujeres debido al intenso dolor, unido a la habilidad y destreza de la persona encargada del corte puede provocar lesiones en la uretra, vagina, perineo $\mathrm{y} / \mathrm{o}$ ano ${ }^{(14)(18)}$.

- Retención urinaria: que puede aparecer a consecuencia al dolor intenso que se produce con la micción o derivado de una inflamación secundaria a una lesión en el conducto uretral ${ }^{(18)}$.

- Muerte: se puede dar como consecuencia de una complicación asociada a otros problemas relativos a la $\mathrm{A} / \mathrm{MGF}^{(3)}$.

\subsubsection{A largo plazo}

Consideraremos como complicaciones a largo plazo aquellas que aparecen en un período de tiempo superior a las diez horas transcurridas desde la operación ${ }^{(14)}$. En algunos casos, los problemas a medio y largo plazo son consecuencia de complicaciones previas que no se han curado o que lo han hecho de una forma inadecuada. Las más características son:

- Anemia severa: la hemorragia producida por la A/MGF unida a situaciones de anemia hereditaria y malnutrición pueda llegar a provocar anemias severas ${ }^{(17)}$.

- Cicatrización anormal(17)(18) :

- Fibrosis: formación patológica de tejido fibroso en los genitales, debido a una cicatrización anormal o cicatrización limitada al sitio de los cortes. 
- Queloides: lesiones de la piel formadas por crecimientos exagerados del tejido cicatricial en el sitio de una lesión cutánea.

- Sinequia: adherencia y fusión anormal que puede ser parcial o total, de los labios mayores o menores.

- Rotación de los tejidos: debido a la pérdida de tejido por una cicatrización anormal y por la retracción de la piel de la zona.

- Incremento de la vulnerabilidad hacia enfermedades de transmisión sexual (ETS). Las condiciones antihigiénicas en las que se suele realizar la práctica; la utilización de cualquier objeto corto-punzante, sin cambiar de una niña a otra de instrumento, y el aumento de la posibilidad de generar heridas por fricción a la hora de la penetración, son varios de los factores que generan un aumento de la vulnerabilidad hacia las ETS.

- Infecciones recurrentes del tracto urinario (ITU): los cambios anatómicos que provoca la $\mathrm{A} / \mathrm{MGF}$ en la vagina y conductos urinarios pueden conducir a problemas en la micción, como dificultad en el vaciamiento de la vejiga debido a la estenosis y/o micción dolorosa, lo que favorecerá la proliferación de microorganismos y como consecuencia infecciones recurrentes del tracto urinario $^{(19)}$.

- Fístulas vésico-vaginales y recto vaginales: en las mujeres "cortadas" se suelen dar como consecuencia de una infección que evoluciona a un absceso y progresivamente a una fístula. También pueden ocasionarse por un acontecimiento traumático durante la intervención.

- Dismenorrea secundaria: trastorno menstrual caracterizado por dolores menstruales severos asociados a alguna complicación de la A/MGF.

Todas las complicaciones anteriormente citadas son comunes a los tres principales tipos de A/MGF, clitoridectomía, escisión e infibulación. La infibulación, al ser la modalidad de A/MGF más cruenta tendrá también una serie de complicaciones añadidas, resultado de la obstrucción mecánica creada por la cicatriz que cubre la uretra y la vagina $^{(14)}$, algunas de ellas son:

- Dismenorrea grave: por la obstaculización del flujo menstrual debido a la angosta salida que queda tras la infibulación.

- Estenosis introito vaginal y vagina como consecuencia de adherencias vulgares.

- Hematocolpos y hematometra: si como consecuencia de la intervención se deja la apertura vaginal demasiado estrecha en una mujer virgen se puede crear una acumulación de sangre menstrual en la vagina y en la cavidad uterina.

- Infecciones pélvicas crónicas. 
- Dificultades a la hora de orinar por la estenosis vaginal creada por la intervención, que puede dar lugar a un estancamiento de la misma tras la cicatriz y crear pequeñas piedras y quistes sebáceos.

\subsection{Obstétrico-ginecológicas.}

En 2006, la OMS ${ }^{(20)}$ realizó un extenso estudio prospectivo sobre un total de 28.393 mujeres, de seis países africanos (Burkina Faso, Ghana, Nigeria, Senegal, Sudán y Kenya), que habían sufrido alguno de los tipos de A/MGF. Este estudio permitió corroborar la existencia de una mayor frecuencia de complicaciones durante el embarazo, en el momento del alumbramiento y en el puerperio en mujeres que han sufrido esta práctica comparadas con las que no la han sufrido. En el estudio también destacan que las complicaciones se dan mayoritariamente en mujeres a las que se les ha practicado la escisión (tipo II) y la infibulación (tipo III). En el estudio se destacaron las siguientes complicaciones obstétrico-ginecológicas:

- Una mayor necesidad de realizar cesárea en el parto y a su vez un mayor índice de hemorragias postparto.

- Una mayor probabilidad de permanecer hospitalizadas durante un tiempo más prolongado que las mujeres que no han sufrido A/MGF.

- Aumenta el número de episiotomías. La episiotomía es una incisión quirúrgica lateral o medial del periné, que se realiza antes de la expulsión de la cabeza del feto, con la finalidad de facilitar la salida del mismo evitando así desgarros en la zona perineal.

- Aumento de la tasa de mortalidad materna.

- El número de neonatos que necesitan reanimación justo después del parto es significativamente más elevado cuando la madre ha sufrido A/MGF.

- La mortalidad perinatal es más elevada en hijos e hijas cuyas madres han sufrido A/MGF del tipo II y III; pudiéndosele atribuir el $22 \%$ de las muertes perinatales de estos/as recién nacidos/as a mujeres con A/MGF.

Otras complicaciones obstétrico-ginecológicas asociadas a la A/MGF y estudiadas en la bibliografía consultada son:

- Un mayor riesgo de infecciones durante el embarazo. El embarazo provoca una serie de cambios fisiológicos en la mujer. Dos de ellos son el aumento de la vascularización de la zona vaginal y la relajación de los músculos lisos del perineo, lo que sumado al hecho de que en las mujeres infibuladas la apertura del canal urinario se mantiene constantemente húmedo, conlleva un aumento del riesgo de infección ${ }^{(21)}$.

- Problemas durante el parto sobre todo en mujeres con A/MGF tipo II y III. El tejido cicatricial derivado de la intervención sobre el perineo de estas mujeres genera una menor elasticidad de la zona, lo que dificulta la realización de un examen vaginal completo para comprobar el progreso del trabajo del parto ${ }^{(21)}$. En las mujeres infibuladas, dar a luz de forma natural es casi imposible debido a que la cicatriz cubre el introito vaginal imposibilitando la salida fetal. La situación es más compleja pues la presión generada sobre el canal del parto por el feto puede derivar en desgarros perineales y hemorragias severas que requieran de intervención quirúrgica ${ }^{(21)}$. 
Además, el trabajo del parto suele ser muy prolongado ${ }^{(22)}$ en las mujeres infibuladas, lo que favorece la aparición de fístulas obstetricias como consecuencia de la presión de la cabeza del feto sobre la pared vaginal y sobre los órganos adyacentes. Por esta razón, a las mujeres infibuladas se les suele practicar la llamada "des-infibulación", consistente en la apertura de la zona suturada, para así posibilitar el parto. Una vez que las mujeres dan a luz, se les vuelve a practicar la infibulación (re-infibulación). Este procedimiento conlleva todos los riesgos de una infibulación inicial, descritas anteriormente, y un mayor número de problemas ginecológicos en el futuro. La des-infibulación y la reinfibulación se suele repetir tras cada parto, ocasionando en la mujer continuas aberturas y estrechamientos de la zona genital. Esto se traduce en la formación de un fuerte tejido cicatrizal que conlleva un aumento de complicaciones en posteriores alumbramientos.

- Complicaciones fetales: la obstrucción, como consecuencia de las características de la región vaginal de las mujeres infibuladas, y el trabajo de parto prolongado pueden provocar asfixia neonatal con sus posteriores repercusiones sobre el neonato como daño cerebral o la muerte ${ }^{(3)}$.

- Infertilidad primaria: los resultados de un estudio realizado por Almorth et al en $2005^{(23)}$, indican una asociación positiva entre la extensión anatómica de la MGF y la infertilidad primaria. El daño en el tejido genital provocado por el corte, con su contaminación microbiana intrínseca, crea un riesgo de infecciones vaginales. Además, en las niñas prepúber no existe un entorno de protección completo contra infecciones, ya que el nivel de estrógenos es bajo, el epitelio de la vagina es delgado y existe una falta de acidez vaginal. Así, en ausencia de este entorno de protección, la infección puede ascender hasta el útero y las trompas de Falopio con el consiguiente riesgo de producir infertilidad primaria. También la alteración de la anatomía normal de la vulva de las niñas puede dar lugar a cambios estructurales y fisiológicos, que a su vez pueden tener efectos negativos sobre la reproducción.

\subsection{Psicosociales.}

Conocer científicamente los efectos psicológicos derivados de la A/MGF entraña una mayor dificultad que los físicos ${ }^{(6)}$. Por ello, para explorar dichos efectos debemos nutrirnos de las experiencias personales de las mujeres afectadas que se atreven a facilitarnos sus vivencias personales de lo sufrido. Así, pese a la falta de pruebas científicas, los relatos personales de mujeres a las que se les ha llevado a cabo la A/MGF revelan sentimientos de ansiedad, terror, humillación y traición, los cuales probablemente tendrán consecuencias negativas a largo plazo ${ }^{(6)}$. Cabe mencionar que los expertos coinciden en que la realización de la práctica unida a todos estos sentimientos, perfilan una personalidad en las mujeres definida como "tranquila" y "dócil", que se traduce en un comportamiento sumiso y obediente considerado como un aspecto positivo de las mujeres en la mayoría de las sociedades donde está afianzada esta práctica, pues hace a las mujeres más "casaderas" por su sometimiento al hombre.

Los sentimientos descritos anteriormente pueden estar negados o mitigados debido a que la A/MGF está vista como algo normal en sus países de origen y se le atribuyen características positivas como orgullo, belleza, limpieza, fidelidad al marido o respeto a su tradición ${ }^{(24)}$. En algunas etnias la realización de la práctica se asocia a 
festividades, regalos y atenciones especiales por parte de la comunidad, lo que genera un sentimiento de pertenencia y aceptación de su sociedad, siendo posible que las mujeres no sometidas a esta intervención puedan sufrir problemas psicológicos derivados del rechazo social y conflictos internos sobre su identidad y lealtad a su cultura y familia.

Por otro lado, las mujeres jóvenes o adultas que han sido sometidas a A/MGF y que luego han emigrado a países occidentales pueden experimentar una serie de problemas psicológicos relacionados con las diferencias existentes entre la cultura del país de origen y la cultura del país anfitrión, dándose cuenta de que la A/MGF no es una tradición universal, si no que está vista de forma muy negativa en otras culturas. Esto puede acarrear graves conflictos internos de identidad y de lealtad hacia su propia cultura, experimentando sentimientos de humillación, confusión, impotencia, sensación de traición de su familia y vergüenza ${ }^{(24)}$. La vergüenza es otro hecho que puede provocar traumas en las niñas o mujeres que han sufrido esta práctica y que viven en un entorno donde además, de estar penalmente castigada es moralmente inaceptable. Esta vergüenza puede llevar a generar problemas de exclusión social y de autoestima.

Asimismo podemos encontrar alusiones, en la bibliografía consultada, sobre la aparición de terrores nocturnos y enfermedades psicosomáticas que generalmente provocan depresión, taquicardias, palpitaciones, dolor u opresión en el pecho, dolores musculares, vómitos, diarrea, etc. ${ }^{(25)}$ También aparece el denominado síndrome de la ansiedad-depresión enfocada genitalmente (Genitally focused anxiety-depression), caracterizado por una constante preocupación de las niñas o mujeres que han sufrido A/MGF sobre el estado de sus genitales y pánico a la infertilidad ${ }^{(25)}$.

Por último, cabe destacar que un estudio de Behrendt y Moritz, en $2005^{(26)}$, muestra la existencia de una prevalencia significativamente mayor de trastorno de estrés postraumático $(30,4 \%)$ y otros síndromes psiquiátricos $(47,9 \%)$ en las mujeres que sufrieron A/MGF en comparación con las mujeres que no la sufrieron.

\subsection{Sobre la sexualidad.}

Los efectos sobre la sexualidad asociados a este tipo de prácticas empiezan a fraguarse desde el momento en el que se lleva a cabo la intervención, aunque las complicaciones respectivas a este apartado suelen evidenciarse en el momento en el que las mujeres inician su vida sexual, normalmente coincidiendo con la noche de bodas. Durante la primera relación sexual, la falta de una educación sexual apropiada y, en los casos de escisión e infibulación, la estenosis del introito vaginal producida por la presencia del tejido fibroso y sin elasticidad consecuencia del proceso de cicatrización, puede ocasionar que el momento del coito sea difícil, traumático y muy doloroso. Por lo tanto, el primer acto sexual sólo puede realizarse tras la dilatación gradual y dolorosa de la pequeña abertura que queda tras la práctica, siendo necesario en algunos casos, la realización de una incisión previa o des-infibulación ${ }^{(6)}$. El dolor durante el coito o dispareunia, no sólo estará presente en la primera relación sexual sino que es posible que se mantenga presente en posteriores penetraciones debido a la poca flexibilidad del tejido cicatricial o a infecciones vaginales recurrentes secundarias. Este dolor puede provocar cierto grado de fobia sexual y trastornos de la excitación. Otra complicación que podemos encontrar en las mujeres que han sufrido A/MGF es el vaginismo, como consecuencia de la dispareunia, y la frigidez asociada con molestias producidas por una infección del conducto urinario o de la vagina 
secundaria a dicha práctica, o a conflictos psicológicos relacionados con las fobias sexuales que causan las penetraciones dolorosas.

En cuanto al placer sexual en estas mujeres puede permanecer intacto, aunque en un gran número de casos suele estar disminuido o abolido. La extirpación de zonas erógenas de gran importancia en la sexualidad femenina, como el clítoris y labios genitales, el tejido cicatricial consecuente y las fobias asociadas al dolor, son algunos de los factores que pueden afectar a la capacidad de excitación y de sentir placer con el coito en estas mujeres. Pueden incluso darse casos de anorgasmia (imposibilidad de sentir placer) ${ }^{(17)}$.

\section{CONCLUSIONES}

- La A/MGF forma parte del sistema de cuidados genéricos tradicionales de las comunidades en las que se realiza, puesto que es entendida como un cuidado cultural de las mujeres determinado por factores socio-culturales, higiénicoestéticos, religioso-espirituales, sexuales y otros relacionados con la salud.

- Las consecuencias sobre las mujeres, derivadas de esta práctica y descritas por la bibliografía, abarcan una amplia gama de complicaciones que van desde las físicas hasta las psicosociales, pasando por las obstétricas y las sexuales. Atentando de esta forma contra la integridad bipsicosocial de las mujeres y niñas a las que se le realiza esta práctica.

\section{BIBLIOGRAFÍA}

- (1) Organización Mundial de la Salud [Sede Web]. Nota descriptiva №: 241, febrero de 2012. Mutilación genital Femenina [Acceso el 28 de noviembre de 2011]. http://www.who.int/mediacentre/factsheets/fs241/es/index.html

Disponible en:

- (2) WHO. Eliminating Female genital mutilation. An interagency statement. [Monography on the Internet]. Geneva: World Health Organizatiin, 2008 [Accessed December 8, 2011]. Available at: http://whqlibdoc.who.int/publications/2008/9789241596442 eng.pdf

- (3) Ramírez Crespo, Mª Victoria y Carmona Rubio, Ana Belén (coord.), La MGF en España. Prevención e intervención [Monografía en Internet]. Madrid: UNAF; 2012 [Acceso el 12 de marzo de 2012]. Disponible en: http://www.africafundacion.org//MG/pdf/MGF en Espana Guia de profesionales. $\underline{\mathrm{pdf}}$

- (4) Kitui, Barbara. Female genital mutilation in South Africa. 2012 June 7. [Accessed June 13, 2012]. In: AfricLaw. Advancing the rule and role of law in Africa [Internet- Blog]. Available at: http://africlaw.com/2012/06/07/female-genitalmutilation-in-south-africa/

- (5) Kaplan Marcusan, Adriana y López Gay, Antonio. Mapa de la Mutilación Genital Femenina en España 2009 [Monografía en Internet]. Barcelona: Servei de Publicacions Universitat Autònoma de Barcelona, 2010 [Acceso el 28 de noviembre de 2010]. Disponible en: http://publicacions.uab.es/pdf Ilibres/AAP0001.pdf

- (6) Amnistía Internacional. La mutilación genital femenina y los derechos humanos [Monografía en Internet]. Edición española, Madrid: Editorial Amnistía Internacional 
(EDAl); 1998 [Acceso el 22 de noviembre de 2011]. Disponible en: http://www.amnistiainternacional.org/publicacion-31

- (7) Bénédicte, Lucas. La ablación genital femenina: una práctica inaceptable desde la perspectiva de los derechos humanos. Balance de la situación y recomendaciones para su erradicación [Tesina], Madrid: Instituto de Derechos Humanos Bartolomé de Las Casas de la Universidad Carlos III de Madrid; 2007.

- (8) Kaplan Marcusan, Adriana y Martínez Bueno, Cristina (coord.), Mutilación genital femenina: prevención y atención. Guía práctica para profesionales. [Monografía en Internet]. Barcelona: Associació Catalana de Llevadoras; 2004 [Acceso el 19 de diciembre de 2011]. Disponible en: http://www.aen.es/docs/guias vg/guia mutilacion genital.pdf

- (9) Bayo, A; González, I; Román, A; Rodríguez, A.B; Grau, S; Marina, J; et al. "La mutilación genital en la mujer del siglo XXI." Progresos de obstetricia y ginecología [Revista on-line]. 2003 [Acceso el 26 de noviembre de 2011], 46(6): 257-66. Disponible en: http://www.elsevier.es/es/revistas/progresos-obstetricia-ginecologia151/la-mutilacion-genital-mujer-siglo-xxi-13048277-revision-2003

- (10) UNICEF. Female Genital Mutilation/cutting. A statistical exploration [Monograph on the Internet]. Ney York: The United Nations Children's Fund, 2005 [Accessed December 8, 2011]. Available at: http://www.unicef.org/publications/index 29994.html

- (11) Kaplan, Adriana y Bedoya, María Helena. "Las mutilaciones genitales femeninas en España: una visión antropológica, demográfica y jurídica" en Javier de Lucas (ed.). Evaluating the impact of existing legislation in Europe with regard to female genital mutilation. Spanish National Report [Monograf on-line]. University of Valencia: 2004 [Accessed January 10, 2012]. Available at: http://www.uv.es/CEFD/17/Daphne SpanishReport February 04.pdf

- (12) Muslim Women's Shura Council. Female Genital Cutting: Harmful and UnIslamic. WISE, in July $2010 . \quad$ Available at: http://www.wisemuslimwomen.org/images/uploads/WISE- Digest.pdf

- (13) Guerra Palermo, María José. "Culturas y género: practicas lesivas, intervenciones feministas y derechos de las mujeres." ISEGORÍA. Revista de filosofía moral y política [Revista on-line]. 2008 enero-junio [Acceso el 22 de noviembre de 2011]; $38 . \quad$ Disponible en: http://isegoria.revistas.csic.es/index.php/isegoria/article/view/403/404

(14) Medicusmundi Andalucía. Mutilación Genital Femenina. Más que un problema de salud [Internet]. Granda: Grupo Editorial Universitario, 2008 [Acceso el 22 de noviembre de 2011] Disponible en: http://www.luchamgf.org/libro.pdf

- (15) Rahman, Anika y Toubia, Nahid. Female Genital Mutilation. A guide to laws and policies worldwide. London: Zedbook, 2000.

(16) Galao Malo, Roberto; Lillo Crespo, Manuel; Casabona Martínez, Isabel; Mora Antón, $\mathrm{M}^{\mathrm{a}}$ Dolores. "¿Qué es la enfermería transcultural? Una aproximación etimológica, teórica y corporativista al término." Evidentia [Revista on-line,] 2005 enero-abril; [Acceso el 4 de enero de 2012]; 2(4). Disponible en: http://www.indexf.com/evidentia/n4/99articulo.php

- (17) Kaplan, Adriana; Hechavarría, Suiberto; y Bonhoure Isabelle. "Health consequences of female genital mutilation/cutting in the Gambia, evidence into action." Reproductive Health [Journal on-line]. 2011 [Accessed December 8, 2011]; 8(26): 1-6. Available at: http://www.reproductive-health-journal.com/content/8/1/26

- (18) Kaplan Marcusan, Adriana; Torán Monserrat, Pere; Bedoya Muriel, María Helena; Bermudez Anderson, Kira; Moreno Navarro, Juani; y Bolibar Ribas, Bonaventura. "Las mutilaciones genitales femeninas: reflexiones para una intervención desde la atención primaria." Aten. Primaria [Journal]. 2006[Acceso el 
4 de enero de 2012]; 38(2):122-6. Disponible en: http://www.mgf.uab.es/docs/MGF Atencion Primaria.pdf

- (19)Almroth, Lars; Bedri, Hibba; El Musharaf, Susan; Satti, Alia, Idris, Taysser; Hasmin, M SIR K; et al. "Urogenital Complications among Girls with Genital Mutilation: A hospital-Based Study in Khartoum." African Journal of Reproductive Health [Journal on-line]. 2005 [Accessed December 8, 2011]; 9(2): 118-123. Available http://www.jstor.org/discover/10.2307/3583468?uid=2129\&uid=2\&uid=70\&uid=4\&si $\mathrm{d}=21100773819181$

(20) WHO. "Female genital mutilation and obstetric outcome: WHO collaborative prospective study in six African countries." The Lancet [Journal on-line]. 2006 